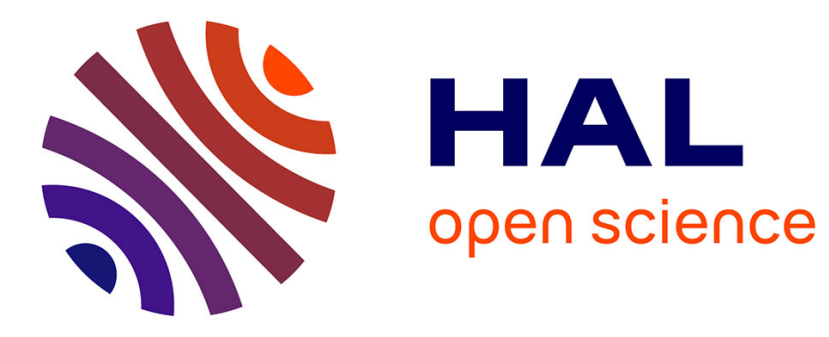

\title{
How to handle the Decision-Maker's awareness of industrial context in his objectives declaration?
}

Jacky Montmain, Lamia Berrah, Vincent Clivillé, Laurent Foulloy, Gilles Mauris

\section{- To cite this version:}

Jacky Montmain, Lamia Berrah, Vincent Clivillé, Laurent Foulloy, Gilles Mauris. How to handle the Decision-Maker's awareness of industrial context in his objectives declaration?. International Conference on Industrial Engineering and Systems Management, escuela superior de ingenieros sevilla spain, Oct 2015, Sevilla, Spain. 10.1109/iesm.2015.7380191 . hal-01224231

\section{HAL Id: hal-01224231 \\ https://hal.science/hal-01224231}

Submitted on 6 Nov 2015

HAL is a multi-disciplinary open access archive for the deposit and dissemination of scientific research documents, whether they are published or not. The documents may come from teaching and research institutions in France or abroad, or from public or private research centers.
L'archive ouverte pluridisciplinaire HAL, est destinée au dépôt et à la diffusion de documents scientifiques de niveau recherche, publiés ou non, émanant des établissements d'enseignement et de recherche français ou étrangers, des laboratoires publics ou privés. 


\title{
How to handle the Decision-Maker's awareness of industrial context in his objectives declaration?
}

\author{
(presented at the 6th IESM Conference, October 2015, Seville, Spain) ${ }^{C} \mathrm{I}^{4} \mathrm{e}^{2} 2015$
}

\author{
Jacky Montmain, \\ LGI2P Ecole de Mines d'Alès \\ Alès, France \\ $\underline{\text { Jacky.montmain@mines-ales.fr }}$
}

\author{
Lamia Berrah, Vincent Clivillé, Laurent Foulloy, Gilles \\ Mauris, \\ LISTIC Université Savoie Mont Blanc \\ Annecy le Vieux, France \\ lamia.berrah@univ-smb.fr, vincent.cliville@univ-smb.fr, \\ laurent.foulloy@univ-smb.fr, gilles.mauris@univ-smb.fr,
}

supplies the expected production by the correct processing of the resources [4].

To be more precise, the Routine situation is implicitly the reference for declaring the objectives, launching the actions, and, more generally, for all the structured decisions that are made. Hence, the risk becomes related to the focalisation on the Routine conditions, forgetting in this sense some kind of awareness of the real encountered conditions. In accordance with the topic of our paper, let us refer to the concept of Situation Awareness (SA), as it has been developed by the Human Factors community in the mid-nineties [5,6]. SA involves becoming aware (i.e. decision-makers and other responsible actors), of all that is happening in the vicinity in order to understand how information, events and one's own actions will alter the objectives achievement, both immediately and in the near future. With a lack, an inadequacy or a weakness, SA has been identified as one of the primary factors in accidents attributed to human error [7]. According to us, the SA generally varies from not-at-all to total, with regards to the encountered situation on the one hand, the concerned DecisionMaker (DM), as well as the evolution of his perception and knowledge about the situation, on the other hand.

Hence, we choose to focus in this study on one particular aspect of management which is the objective declaration. We look for a type of handling of the soundness of such a declaration with regards to industrial encountered situations on the one hand and to the SA of the Decision-Maker (DMSA) on the other hand. To be more precise, the objective declaration is generally made by a DM, who has the authority and the control of the considered business unit or physical system that is concerned with the achievement of the objective. Moreover, knowing that an objective is declared by a target value and a temporal horizon $[4,8,9]$, both these parameters of the objective have to be, from a technical point of view, realistic with regards to the achievement. They also have to, from a strategic point of view, make the performance perennial. Hence, the DM becomes responsible for what can be called the soundness of the objective declaration. However, declaring accurate and relevant context-dependent objectives is thornier. A reliable knowledge and a strong SA thus allow the DM to first be able to identify the right situation and then deduce the right objectives values from this diagnosis. 
Hence, the achievement of the initial declared objectives becomes more or less relevant/certain, depending on the current situation and leads thus to either the impossibility of performing, or bad performances. The purpose of this work is to adapt objectives declaration to the real situation that is encountered by the company or the physical considered system. Beyond the Routine situation, three situations are then considered: the Control situation, the Emergency Crisis situation and the Loss of Control situation. We suggest more particularly that such adaptation depends on the industrial situation, and that the characterisation of the industrial situation is related to the DMSA. That is why we look to handle the DMSA in the objective re-declaration mechanism, assuming in this sense that the DM is more or less aware of what is happening in the current situation. We imagine that the DM becomes more or less able to give relevant pieces of information about this situation, and, consequently, is more or less able to re-declare relevant values for the objectives. Moreover, we assume that the DMSA may evolve, and generally increase, by the occurrence of internal or external events that may impact on the DM's way of thinking. The developed idea here is then the modelling of such awareness by a triplet that involves the Belief of the DM on what he gives in terms of knowledge, his ability to characterise the Level of the situation as well as its Graduality. We assume moreover that the DMSA is something which needs to be captured, and types of decision-aiding systems can be useful in this sense.

Beyond the quantification of the DMSA but also depending on it, the situation is described through parameters such as, at minima, its name, and possibly the quantification of its level, the grade of membership or characterisation associated with it... The objective is then re-declared according to the expressed DMSA and the encountered situation characterisation. Mathematical translations and thresholds are thus proposed in order to obtain the re-declared values.

This paper is thus organised as follows. After a presentation of both the characteristics of the industrial context and its four typical situations, and the SA concept in Section 2, we introduce, in Section 3, a parametric way of quantifying the DMSA concept as well as a methodology for capturing such a SA. After a brief recall of the objective declaration principle, some examples of objectives re-declaration are then presented, on the basis of numerical initial declared objectives. We give after, in Section 4, illustrations that are extracted from a steel manufacturer. Concluding remarks will thus emphasise the prospects of this study.

II. SOME ELEMENTS CONCERNING THE INDUSTRIAL CONTEXT SITUATIONS AND THE DECISION-MAKER AWARENESS

\section{A. The industrial context situations}

According to the industrial management literature, four situations are considered as identifying the context of industrial systems (see Fig. 1) [3]. These situations are defined according to, on the one hand, the ability of the operational system to normally operate and, on the other hand, to a kind of control degree, by the DMs, of the considered systems.

By considering Fig. 1, one can see that the reciprocal function pairs (Control versus Emergency Crisis) and (Routine versus Loss of Control) are generalised, for example as respectively "incentives to action" and "incentives to inaction", with regards to the considered objectives. Control consists of acting to reach a desired state or event, namely a revisited

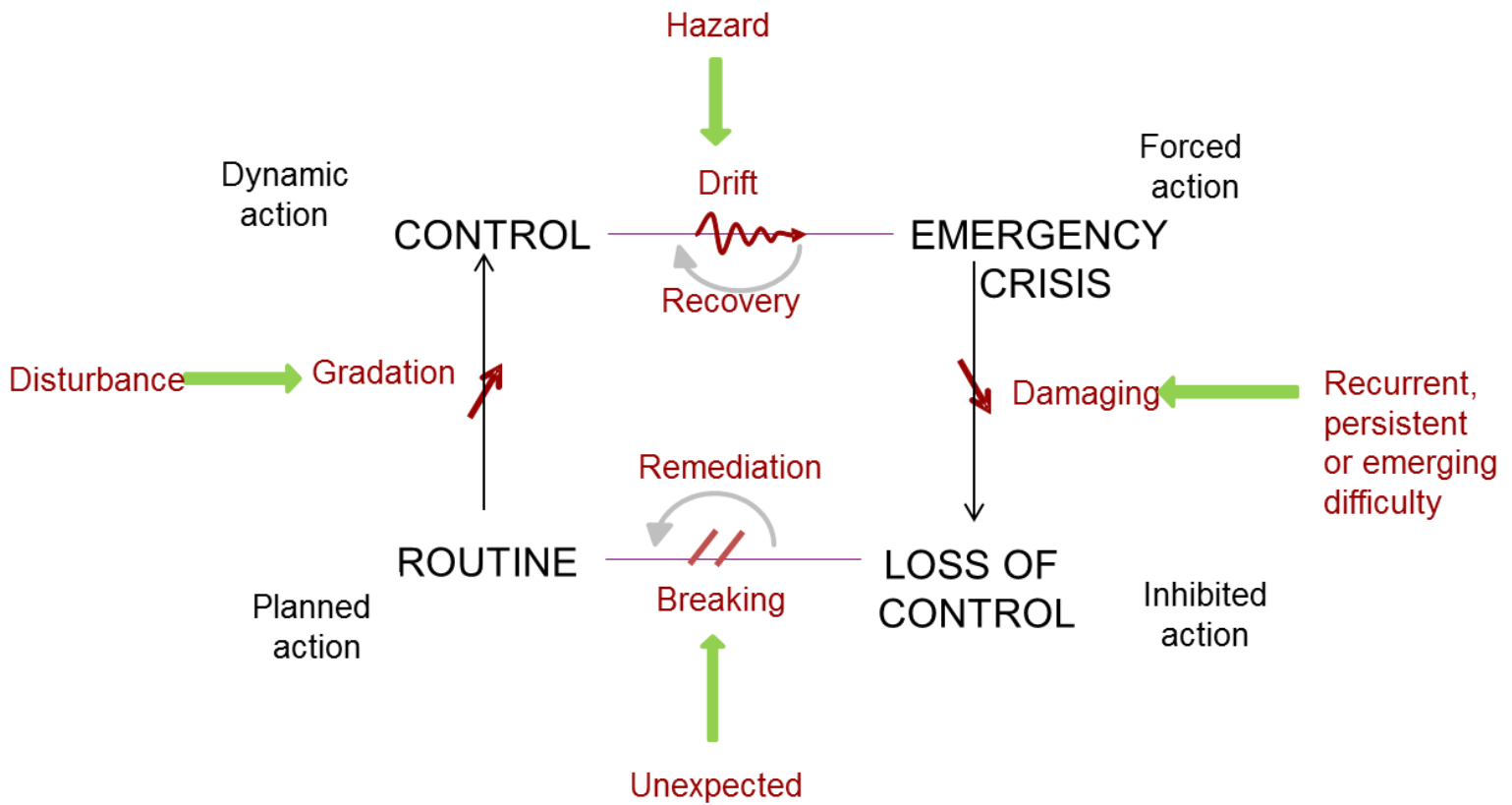

Fig. 1. Industrial management context 
initial objective. Emergency Crisis involves opposing an undesired state or event. Routine and Loss of Control both consist of doing nothing in particular, because action is either unnecessary or impossible. The Emergency Crisis situation may be considered as the situation which is thornier to be correctly perceived because of its un-foreseeability (unlisted events, data transmission errors, events misunderstanding...) and that the reaction is inappropriate. Loss of Control reflects a situation in which it is impossible to implement coherent action with the objectives.

In Control situations that arise when unexpected events do appear, actions and decisions imply sophisticated strategies to face threatened incidents and unforeseeable consequences. Building a representation of such situations and planning actions are thorny tasks because of the uncertain environment and the unpredictability of phenomena that cannot be wholly modelled. In these situations, the organisation does the best it can to avoid threatened incidents and progressively improve the business unit functioning.

Emergency Crisis situations appear when threatened incidents occur or at least are pending. They can no longer be avoided. DMs have much less flexibility as in previous situations because the activity is already suffering damageable consequences. Decisions then aim at reducing consequences of threatened incidents on the activity, staff and environment.

Decision and action can still be inhibited in Loss of Control situations when DMs are faced with unthinkable situations.
Such situations had never been envisaged in the framework of the company activity. Note that unpredictability can be caused by inadequate action plans with regard to the situation, by ineffective action plans, etc. In such cases, surprise appears as the crisis factor. Finally, Loss of Control situations can be the result of the deterioration of Emergency Crisis situations, when resources cannot prevent the disaster any longer or disturbances become recurrent. The system is destabilised and uncertainty is maximal.

Let us note that in this study only a few generalities are given, and that it is for introducing the four situations. For further information, one can consider $[3,10,11]$.

\section{B. The Situation Awareness SA}

SA-related cognitive mechanisms (perception, comprehension, projection into the future), decision-making and performance assessment are all embedded into a cognitive feedback loop (see Fig. 2) [5,6].

The perceptual level 1 involves the detection, recognition and identification of elements that define a specific situation. Perceptual SA relies on available sensory information (e.g. from sensors in control situations, economic indicators in management situations) as well as the operator's/DM's prior knowledge (e.g. object patterns/diagrams activated by memory) in order to identify individual situation elements and object groups. Comprehension level 2 reflects an understanding of the current state of affairs and involves

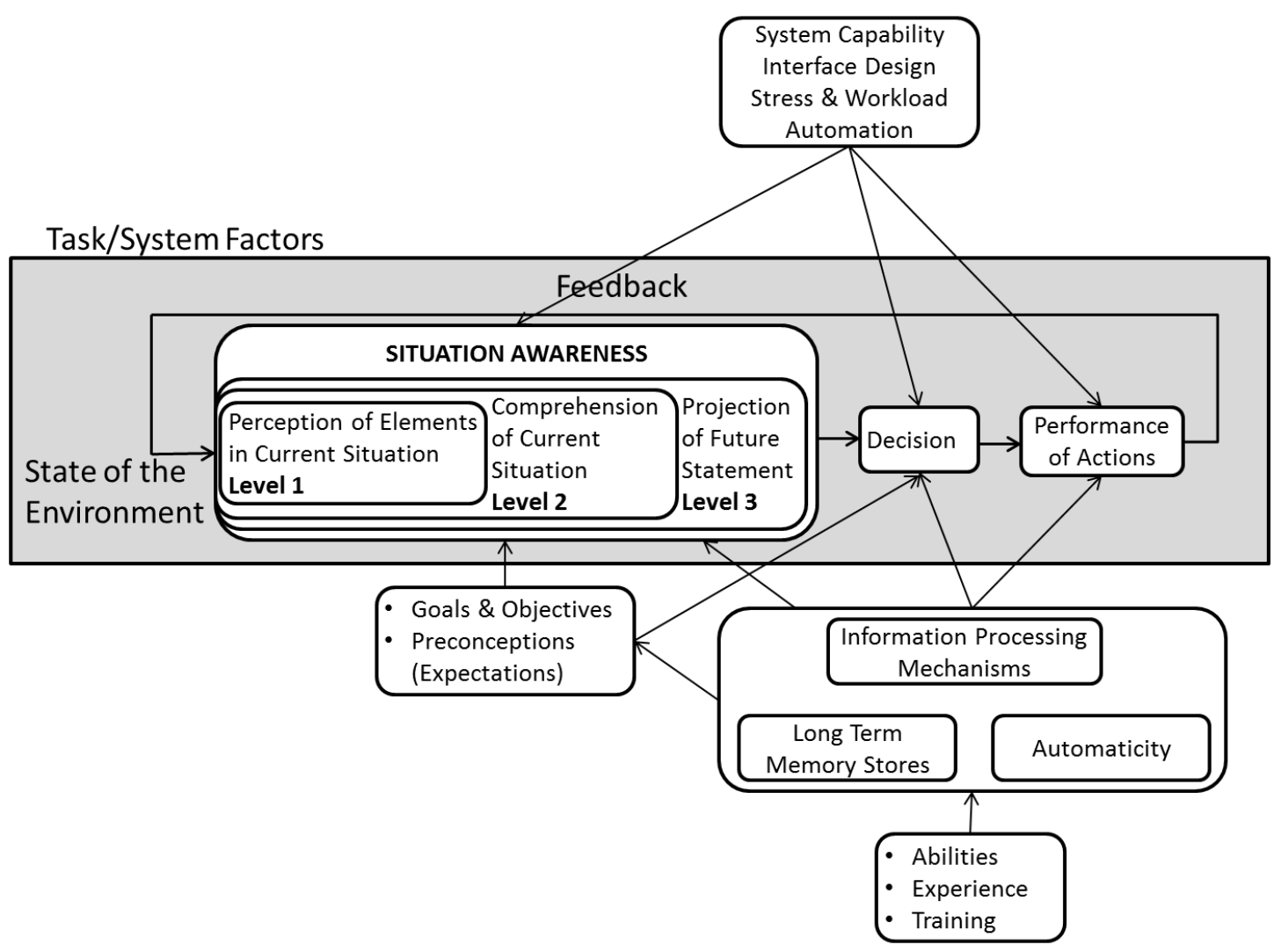

Fig. 2. Situation Awareness and Decision-Making [6]. 
making inferences about activities in the current situation. As such, the comprehension level maps perception products into object functions. Finally, projection level 3 consists of interpretations concerning the trajectory of the situation based on the output of Comprehension SA and prior knowledge [12].

Note that, for the sake of simplicity, we are looking here to handle each SA level (Perception, Comprehension, Projection) by one parameter, by looking for some kind of elicitation and quantification of it as it is described in the next section.

\section{RE-DECLARING THE OBJECTIVES}

\section{A. Some indications with regards to the DMSA capture}

DMSA capture is one of the key points of our approach. As has been seen above, the DMSA concept handles the awareness of a DM with regards to an industrial encountered situation. Hence, we choose to consider the DMSA using three parameters. Even if these parameters are associated with the $\mathrm{DM}$, the first one is related to his perception of the situation (SA Level 1) while the two others are related to his ability to characterise the occurred situation (Comprehension SA Level 2) and its possible evolutions (Projection SA Level 3) (see Fig.2). Namely, we have:

- the Belief, which is the ability of the DM to evaluate the truth of his knowledge about the situation;

- the Level, which is the ability of the DM to quantify a level in a given situation;

- the Graduality, which is the ability of the DM to qualify the situation with a membership grade.

Note that the Graduality parameter intervenes only when the system is changing and the DM is facing a transition between two situations. When only one situation is identified, the Graduality is naturally not taken into consideration.

Representing each criterion on a $[0,1]$ scale leads to the Awareness Unit Cube (AUC) represented in Fig. 3.

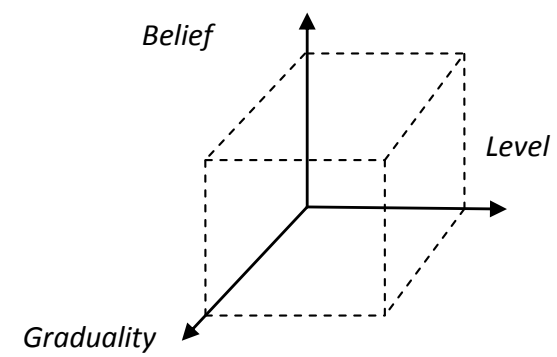

Fig. 3. The Awareness Unit Cube

Let us represent the vertices as BLG coordinates, i.e as respectively Belief, Level, Graduality vectors. Let us note that vectors are expressed as BLG coordinates while the DM decided to represent the Belief on the conventional z-axis. By doing so they wanted to emphasize the importance of belief increase as an elevation.

Vertices such that the Belief is equal to 0, i.e. [ $\left[\begin{array}{lll}0 & 0 & 0\end{array}\right]$, $\left[\begin{array}{lll}0 & 1 & 0\end{array}\right],\left[\begin{array}{lll}0 & 0 & 1\end{array}\right]$ and $\left[\begin{array}{lll}0 & 1 & 1\end{array}\right]$, correspond to DM whose situation analysis can hardly be trusted. Vertices such that the Belief is equal to 1 correspond to DM who is confident in the qualification and/or quantification of the situation. The Belief parameter becomes the most impacting one. A high level of Belief is thus a requirement to trust information on Level or Graduality.

We propose to differentiate some typical DMSA with several $*$. The greater number of $*$, the better in terms of situation characterisation.

- The vertex [lll 100$]$ is associated to $1 *$-DM. DM has a total Belief with regards to the situation he is facing, namely: Routine, Control, Emergency Crisis, Loss of Control (\$II.B).

- The vertex [ $\left[\begin{array}{lll}1 & 1 & 0\end{array}\right]$ is associated to $2 *-\mathrm{DM}$. The DM is able to qualify not only the situation but also its Level. The quantification can either be numerical, e.g. Emergency Crisis with a Level 5 which means that the DM has a total ability to give the Emergency Crisis situation Level, and gives, for instance, the " 5 " value to it. The quantification can also be linguistic, e.g. a Severe Emerging Crisis, which means that the DM has a total ability to give the Emergency Crisis situation Level, and gives, for instance, the "Severe" value to it.

- The vertex [ $\left[\begin{array}{lll}1 & 0 & 1\end{array}\right]$ is associated to $3 *$-DM. The DM is, moreover, able to give the Graduality of the encountered situations. For instance, the DM is able to consider that the situation can be Routine with a certain grade of membership, e.g. 0.6/Routine - that is Routine with a grade of membership equal to 0.6 - but also Control or Emergency Crises to different grades of membership.

- The vertex [ $\left[\begin{array}{lll}1 & 1 & 1\end{array}\right]$ is associated to $4 *$-DM. In this case, the DM has a total SA, based on his Belief as, e.g., "I believe that the situation is 0.6/Emergency Crisis. In that case, I consider the Emergency Crisis as Very Severe. But the situation could also be 0.2/Loss of Control. And in that case, I consider that we are faced with a Medium Loss of Control situation."

Let us note that this typology corresponds to crisp cases that are linked with the vertices of the UAC (see Fig. 3). In the general case, one can imagine that the DMSA is a point in the UAC. This point in then defined by its BLG coordinates. Moreover, one can imagine also that the DMSA can evolve in time, according to the increasing perception level of the DM. In this sense, the DMSA becomes a non-static notion. Many parameters can make the SA of the DM change, such as the occurrence of some events, the analysis of what is happening, some oriented discussions with experts... Our aim is to make the DM increase his SA. Let us recall that with a better SA, the DM will better characterise the encountered situation. He will then be able to re-declare the objectives in a relevant manner. We propose here to proceed to a questioning of the DM, in such a way that answering our questions makes him become more and more aware of what the situation is. We assume, as a starting point for the DMSA capture, the worst case, namely 
the case where the DM has no SA. This starting point is the origin of the UCA, i.e. the BLG vector is $\left[\begin{array}{lll}0 & 0 & 0\end{array}\right]$. In this logic, the ideal ending point is when the DM becomes a $4 *-\mathrm{DM}$, namely with a BLG vector [ $\left[\begin{array}{lll}1 & 1 & 1\end{array}\right]$, having thus a total SA. The time-dependent trajectory in the UCA between these two points represents the DMSA evolution.

In our vision, this DMSA capture process relies on several structured steps.

- The first one consists of working on the Belief axis. Indeed, the DM has to be able to have a strong Belief in his characterisation of the occurred situation.

- When this step is achieved, the DM is close to a $1 *$-DM. Then, the Level axis can be considered such that the DM becomes aware of the Level of the encountered situation.

- When this step is achieved, the DM is close to a $2 *-D M$. Finally, the Graduality axis can be considered to drive the

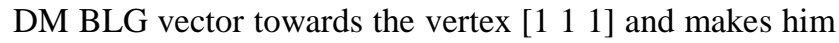
a $4 * D M$.

Note that we assume that the SA capture is made independently from the encountered situation. The questions asked to the DM are oriented towards some generic parameters that may allow him to be aware of what is happening.

\section{B. The objective re-declaration mechanism}

Let us recall that an objective is considered through a target value to achieve at a given time $[8,9,13]$. The value as well as the temporal horizon is declared with regards to a considered variable [9]. Such a variable is generally associated with the actions to launch in order to achieve the objective. Previous works have dealt with the definition of a unified framework for handling several kinds of declarations; such as numerical, linguistic, crisp or imprecise... [9,14]. Moreover, the objective declaration concerns the step where the DM gives this value. According to our classification of the situations, the objective declaration corresponds to the Routine situation and, to the best of our knowledge, very few works have been published concerning industrial objectives declaration in other situations that the Routine situation (§II.A). Even if these situations are less frequent and could be separately addressed, our proposal is to consider them in the unified framework of the objective redeclaration, leading thus to analyse, in further works, the transitions between these situations.

Re-declaring the objective consists thus of changing the objective value according to the DMSA first and, secondly, the characterisation of the encountered situation. The basic ideas are to obtain fewer and fewer challenging objectives as the severity of the situation increases. Obviously, the more aware the DM is, the sounder the re-declaration of the objective. For the sake of simplicity, we consider an objective such as the greater its value, the greater the DM satisfaction with regards to its achievement. Moreover, we choose to focus here only on the value changes, leading thus to maintain as constant the corresponding temporal horizon. For instance, whatever a characterised DMSA, we can imagine the following rules.

- In the Routine situation, the objective value is not modified.
- In the Control situation, the objective value is reduced.

- In the Emergency Crisis situation, the objective value is reduced and some imprecision in its declaration is possible. Thus the aim is to stop the drift that causes the disturbed situation and recover as soon as possible a control situation (see figure 1). The emergency and damage characteristics of the crisis situation prevent managers to fit precise objective because they are faced with a management situation they are not used to run. It corresponds to knowledge and responsibilities outside of their traditional purview.

- In the Loss of Control situation, the objective value is reduced with a high imprecision degree, leading thus to a translation of the value into a satisfaction (i.e. performance) expression function.

These ideas are emphasised in Fig. 4. We consider the case of a numerical objective value, where $w, x, y, z$ are real values. Besides, $m$ is the variable related to the measurement which is associated with the objective achievement [14]. Simple bounded values are given for the three first cases, while a function is proposed with regards to the fourth case.

Depending on the DMSA, the general previous principles can be refined. As an illustration, let us consider a refinement scenario in the case of the Control situation, assuming once again a numerical objective value. In the same previous assumptions with regards to the objective declaration and satisfaction, the general principle is to re-declare the objective value such that the new objective value is lower than the Routine objective value. Possible refinements are given in the following.

- $\quad 1 *-\mathrm{DM}$ is able to provide re-declaration such as "reduction of the objective by $5 \%$ " which leads to $y=0.95 z$

- $2 *$-DM provides the same re-declaration as $1 *_{\text {- }}$ DM, but also defines a computation rule in order to take into account the Level in the Control situation, by considering, for instance, a linear function.

- $\quad 3 *$-DM provides the same re-declaration as $1 *$ DM, but also defines a computation rule to take into account the Graduality of the situation, by using, for instance, a weighted mean.

- $4 *-D M$ provides the same re-declaration as $2 *$ DM and $3 *$-DM. 


\begin{tabular}{|c|c|}
\hline Routine & Control \\
Objective $=z$ & Objective $=y<z$ \\
\hline Emergency & Loss of Control \\
\hline $\begin{array}{l}\text { Satisfaction function } \\
\sqrt{[w, x]} \mathrm{y} z\end{array}$ \\
\hline
\end{tabular}

Fig. 4. The re-declaration values for a numerical objective

To summarise, one can say that in the general case, the re-declaration of the objective value is a more or less complex function which depends on:

- the encountered situation;

- the DMSA, i.e. the BLG vector;

- the characterisation of the encountered situation, by pieces of information that are provided by the DM.

Let us specify once again that, in this framework, the DM characterises the situation, according to his SA. The DM may hence give pieces of information such as the quantification of the Level, the grades of membership, the DM Beliefs.

Now, before describing the steel manufacturer case study, let us introduce some necessary notations, according to our previous developed framework [9]. When the DMSA is totally lacking, no information is assumed to be available concerning the encountered situation. Hence, the objective declaration is denoted $o(v)$ where $v$ is a variable under consideration, e.g. $o$ (LotSize). When the objective is re-declared by a $1 *$-DM, the situation is introduced leading to $o(v, s)$ where $s$ is the situation, e.g. o(LotSize, Routine). The re-declaration by a $2 *_{-}$ DM requires the introduction of the quantification of the Level, denoted $l$, leading to $o(v, s, l)$ as for example, $o$ (LotSize,EmergencyCrisis,Severe). In the same manner, other parameters like the grades of membership can be introduced for the re-declaration by a $3 *$-DM or a $4 *$-DM.

\section{CASE STUDY}

\section{A. Presentation}

Let us illustrate our proposition by considering a steel manufacturer which produces black coils and sheets with a capacity of four million tons per year. The means of production of the plant are continuous processes, including blast furnaces, oxygen converters, continuous casters, one hot strip mill and six finishing lines. The main customers of the company are the automotive industry, the shipyards, the heavy-metal...
Incidentally, the steel production is strongly sensitive to the demand, and the demand depends on the classical industry cycles of ten years [15]. Usually, the company is in work, in a continuous manner, for about 150 hours per week, according to the work load, as is planned by the MRP. Four types of products are manufactured, respectively black coil, pickled and oiled coil and sheet, cut to length sheet, slit coils. Each kind of product is proposed in several thicknesses, widths and finishings (surface aspects). The daily production is about 10000 tons. The production is defined with regards to the long term forecasts, and inventories allow the DMs to face the possible order variations. So the manufacture looks for economic lot sizing that involves both important work in progress and inventories.

This way of managing production was satisfying in a Routine context, where the demand variations were not very fast and the competition conditions with the other steel producers were relatively well known and controlled. However, during these last ten years, many events have occurred, disturbing the factory's activity. Namely, a fusion with another company has been effected. Moreover, the recession of 2008 has impacted the performance of the company and, on a tactical level, internal reengineering has modified the production plant functioning. Adding to these points, the manufacturer has observed the competition of new steel actors as well as demand evolution (new finishings for instance), geographical location of the main customers (more and more of them are located in Asia or South America), and, on the operational level, incidents related to the use of some obsolete means of production.

The consequences of these diversified events were a chaotic evolution of the inventories. There were both numerous stock outs with regards to certain references, on the one hand, and very high levels of inventory on other references, on the other hand.

In this context, discussions have been undertaken with the plant head manager - the considered DM in this case - in order to help him to take into account the particularities of the encountered situation and the involved changes, for making his decision, especially for declaring and re-declaring his manufacturing objectives. To be more precise, the submitted problem was related to the adjustment of the lot sizing according to the demand and the finish line flexibility.

Taking into account such particularities and changes require the capture SA of the DM. That is what we have begun to do, according to pre-established awareness capture questionnaires.

\section{B. The DMSA capture}

The plant activity has known many situations changes, with regards to the long temporal horizon of ten years on the one hand and to important number of events that have been encountered, on the other hand. Thus, we have distinguished, during our study, several periods in the lead discussions and questionnaires. These periods are coherent with the evolution of DMSA and its capture. The starting point was corresponding to no SA for the DM. The ending point 
correspond to a SA that allows the DM to characterise not only the situation but also its Level. The DM became then almost a

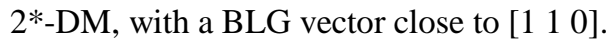

\section{1) Period 1 - the DM has no $S A$}

Before the beginning of this work, the DM was lacking from SA. In that sense, his BLG vector was equal to [l 000 .

\section{2) Period 2 - the DM becomes a $1 *_{-} D M$}

Based on his long experience in the steel industry, the DM was able to easily answer the questionnaire, highlighting thus his SA. The DM was able to qualify and characterise the plant situation, using the four typical situations scale, namely the Routine, the Control, the Emergency Crisis and the Loss of Control (§II.A). It was easily agreed that the Routine corresponds to the conventional regular situation that is associated with the first period. For instance, the Emergency Crisis qualifies the situation at the end of 2008 when the demand of the automotive sector fell by more than $50 \%$, while the Control situation was related to the unusual problem of quality and maintenance which significantly worsened the line productivity. The DMSA was characterized by a BLG vector very close to $\left[\begin{array}{lll}1 & 0 & 0\end{array}\right]$ and the DM was rated as a $1 *$-DM.

\section{3) Period 3 - The DM becomes a $2 *-D M$}

After some weeks of discussions, the DMSA continued to increase. The DM was able to provide more explicit information concerning the situation and became aware of the situation Level. He was able to qualify this level by a relevant additional word among a set of possible words. For instance, the Control situation has been qualified as Partial, Intermediary or Total. Thus the DMSA was characterised by a vector close to [ $\left[\begin{array}{lll}1 & 1 & 0\end{array}\right]$ and the DM was tending towards a $2 *_{\text {- }}$ DM.

For the sake of simplicity, let us note that the last dimension of the SA, i.e. the Graduality between typical situations, has not been considered in this study.

For each period, the objective re-declaration was analysed with the DM, according to the approach which is summarised in Fig. 4.

\section{Objective re-declaration by a DM with no $S A$}

No re-declaration is required in this case. The lot sizing objective was 2 days of production with 10000 tons/day for the finish line. Formally, the declaration is $o($ LotSize $)=20000 \mathrm{~T}$.

\section{Objective re-declaration by a $1 *_{-}-D M$}

\section{a) Routine}

In the Routine situation no objective re-declaration is needed and the objective defined when the DM was with no $\mathrm{SA}$ is kept. Formally, the situation is introduced in the objective leading to $o($ LotSize, Routine $)=20000$ r .

\section{b) Control}

In this situation, the DM proposed to decrease the Routine objective value in order to avoid the saturation point where the production will get worse. As explained before (§III.B), the corrected objective should be such that $o$ (LotSize, Control) $<$ o(LotSize, Routine) . In our considered case, it leads to a reduction of the production by $2000 \mathrm{~T}$, i.e. $o($ LotSize, Routine $)=18000 \mathrm{~T}$.

\section{c) Emergency Crisis}

In this situation, the DM proposed to reduce the Routine objective in order to permit a better reaction to the demand as well as a better correction of the line deviation due to major breakdowns or quality problems. The correction became:

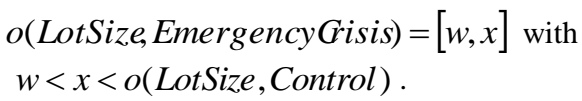

After discussion, it was proposed to consider the interval $[w, x]=[8000,16000]$.

\section{d) Loss of Control}

In this situation it is difficult for the DM to give pieces of information about the correction of the initial declared value. It is a very unusual situation which is, by nature, unpredictable. Finally, the DM agreed with our proposition to consider the objective as a satisfaction function. Let $p$ be a value in $[0,1]$ reflecting the DM satisfaction. $p=0$ means a total dissatisfaction and $p=1$ a total satisfaction. It was proposed to define two values, $a$ and $b$, respectively corresponding to objectives where $p=1$ and $p=0$. Between these values, the DM satisfaction is linearly varying. It led to:

$$
o(\text { LotSize,LossOfConrol, } m)=\left\{\begin{array}{l}
0 \text { if } m<a \\
\frac{m-a}{b-a} \text { if } m \in[a, b] \text { and } \\
1 \text { if } m>b
\end{array}\right.
$$$$
[a, b]<o(\text { LotSize, EmergencyCrisis })^{l} \text {. }
$$

The DM considered that the sustainability of the company could not be guaranteed if the main customer requirement could not be satisfied which gave the lower bound $a$. It was also considered that, in this situation, delivering the three main customers was, at least, what could be done to be satisfied, thus proving the upper bound $b$. Thus, the values $a=4000 \mathrm{~T}$ and $b=7000 \mathrm{~T}$ have been chosen.

$$
{ }^{1} \text { Let } U=[a, b] \text { and } V=[c, d]
$$


Preliminary assumptions and limits have been expressed here, with regards to the kind of decision, on the one hand, and the context of decision-making, on the other hand. More particularly, the considered decision was concerned with objective declaration and adjustment. And the context of decision-making was handled by four classical situations that are the Routine, the Control, the Emergency Crisis and the Loss of Control. Objectives are generally declared with regards to normal Routine situation. Thus the aim of this study was then to look for the soundness of the objectives declaration whatever the occurred situation.

The developed idea is simple. With regards to the considered situation, the objective value is re-declared, the redeclared value being adjusted to the real encountered situation and conditions. Namely, the DM characterises the encountered situation and according to this characterisation, re-declares the objective. Mathematical operations can be used in this sense. However, if the idea is simple and almost classic, the novelty of the work is in the establishment of a link between the DM situation awareness - DMSA -and the way that he characterises such a situation. Some definitions and rules have been given in order to formalise this link. The AUC - Awareness Unit Cube - has been thus introduced, as a basis for the DMSA evolvement capture.

This article represents a first step in the investigation of the handling of the DM behaviour. Many axes can be envisaged in order to enrich these thoughts. Transitions between situations have to be considered as well as the handling of the sincerity and relevance of the DM when he estimates his SA. Qualitative and imprecise objectives can be imagined and redeclared using fuzzy mechanisms... Further developments are in progress in this sense.

\section{REFERENCES}

[1]

2012

R. W. Griffin, "Management", Cengage Learning editor, 720 p. ,

[2] Weihrich and Koontz, "Management: A Global Perspective", McGraw-Hill Education, 640 p., 2004.

[3] J. Montmain, and J. Penalva, "Operators aids: automation and supervision. 9th symposium on information control in manufacturing", INCOM'98, Nancy-Metz, France, 1998.

[4] Y. Ducq, D. Chen and G. Doumeingts, "A contribution of system theory to sustainable enterprise interoperability science base", Computers in Industry, 63(8), pp. 844-857, 2012.

[5] M.R. Endsley, "Measurement of situation awareness in dynamic systems", Human Factors, 37(1), pp. 65-84, 1995.

[6] M.R. Endsley, "Toward a theory of situation awareness in dynamic systems", Human Factors, 37(1), pp. 32-64, 1995.

[7] C.E. Hartel, K. Smith and C. Prince, "Defining aircrew coordination: searching mishaps for meaning.", 6th Symposium on aviation psychology, Colombus, Ohio, 1991.

[8] L. Foulloy, V. Clivillé and L. Berrah "Fuzzy interpretation of performance scorecards for decision-making in the industrial context", Proceedings of the 19th World Congress IFAC (International Federation of Automatic Control), Cape Town, South Africa, 6p, 24-29 August 2014.
[9] L. Berrah and L. Foulloy, "Towards a unified descriptive framework for industrial objective declaration and performance measurement", Computers in Industry, 64(6), pp.650-662, 2013.

[10] A. Imoussaten, "Modélisation et pilotage de la phase de délibération dans une décision collective : vers le management d'activités à risques. " $\mathrm{PhD}$ thesis, Ecole Nationale Supérieure des mines de Paris, in french, 2011.

[11] C. Sanchez, "Spécification et Implémentation du Système d'Aide à la Décision Multicritère pour la Maintenance Préventive et la Gestion du Patrimoine de la Société d'Autoroute ESCOTA : le projet SINERGIE", PhD thesis, Ecole Nationale Supérieure des mines de Paris, in french, 2007.

[12] J.M. Curiel, M.D. Anhalt, J.J. Tran and K-T. Yao, "Developing Situation Awareness Metrics in a Synthetic Battlespace Environment", Interservice/Industry Training, Simulation, and Education Conference (I/ITSEC), Orlando, Florida, 2005.

[13] Y. Ducq, B. Vallespir and G. Doumeingts, "Coherence analysis methods for production systems by performance aggregation”, International Journal of Production Economics, 69(1), pp. 23-37, 2001.

[14] L. Berrah, G. Mauris, A. Haurat, and L. Foulloy, "Global vision and performance indicators for an industrial improvement approach", Computers in Industry, 43(3), pp. 211-225, 2000.

[15] H. Tan and J. A. Mathews, "Identification and analysis of industry cycles", Journal of Business Research, 63(5), pp. 454-462, 2009.

[16] E. Ruspini, "A new approach to clustering", Information and Control, 15(1), pp. 22-32, 1969. 
